\title{
Estratégia Saúde da Família, saúde suplementar e desigualdade no acesso à mamografia no Brasil
}

\author{
Antônio Carlos Vieira Ramos, ${ }^{1}$ Luana Seles Alves, ${ }^{1}$ Thaís Zamboni Berra, ${ }^{1}$ \\ Marcela Paschoal Popolin, ${ }^{2}$ Marcos Augusto Moraes Arcoverde, ${ }^{3}$ Laura \\ Terenciani Campoy, ${ }^{1}$ José Francisco Martoreli Júnior, ${ }^{1}$ Luís Velez Lapão, ${ }^{4}$ \\ Pedro Fredemir Palha ${ }^{1}$ e Ricardo Alexandre Arcêncio ${ }^{1}$
}

Como citar Ramos ACV, Alves LS, Berra TZ, Popolin MP, Arcoverde MAM, Campoy LT, et al. Estratégia Saúde da Família, saúde suplementar e desigualdade no acesso à mamografia no Brasil. Rev Panam Salud Publica. 2018;42:e166. https://doi.org/10.26633/RPSP.2018.166

RESUMO Objetivo. Avaliar a associação entre o acesso à mamografia no Brasil e a cobertura pela Estratégia Saúde da Família (ESF) e pela saúde suplementar.

Métodos. Realizou-se um estudo ecológico com dados obtidos do Departamento de Informática do Sistema Único de Saúde (DATASUS). A tendência da série temporal foi analisada pelo método de Prais-Winsten utilizando-se como unidades de análise as unidades federativas brasileiras. Para investigar a relação entre a variável dependente - mulheres de 50 a 69 anos que nunca realizaram exame de mamografia - e as independentes, de cobertura de ESF ou saúde suplementar e socioeconômicas, realizou-se análise de regressão linear múltipla.

Resultados. O Acre foi o único estado que não apresentou tendência crescente da cobertura da saúde suplementar. Roraima, Tocantins, Maranhão, Piauí, Rio Grande do Norte e Paraíba apresentaram tendência estacionária para a cobertura pela ESF, enquanto as demais unidades federativas apresentaram cobertura crescente. Observou-se associação significativa entre nunca ter realizado mamografia na idade de 50 a 69 anos e as variáveis renda média per capita e cobertura pela ESF e saúde suplementar $\left(R^{2}=0,77\right.$; $\mathrm{P}<0,001$ ).

Conclusão. A desigualdade no acesso a mamografia é uma realidade no Brasil. Tanto a saúde suplementar quanto a Estratégia Saúde da Família têm contribuído para melhoria do acesso dessas mulheres.

Palavras-chave Atenção primária à saúde; Estratégia Saúde da Família; saúde suplementar; mamografia; desigualdades em saúde; Brasil.

\footnotetext{
Universidade de São Paulo (USP), Escola de Enfermagem de Ribeirão Preto, Ribeirão Preto (SP), Brasil. Correspondência: Antônio Carlos Vieira Ramos, antonio.vieiraramos@outlook.com

2 Universidade Federal de Mato Grosso (UFMT), Instituto de Ciências da Saúde, Campus de Sinop, Sinop, (MT), Brasil.
}

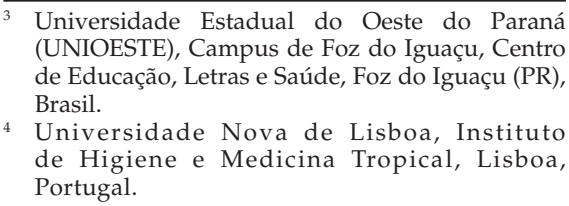

3 Universidade Estadual do Oeste do Paraná (UNIOESTE), Campus de Foz do Iguaçu, Centro de Educação, Letras e Saúde, Foz do Iguaçu (PR), Brasil.

4 Universidade Nova de Lisboa, Instituto de Higiene e Medicina Tropical, Lisboa, Portugal.

O câncer de mama é a segunda neoplasia que mais mata mulheres no Brasil, com 57960 novos casos e 14388 mortes por esse câncer em 2016 (1). Por trás desses números estão grandes desigualdades que têm caracterizado a saúde no 
Brasil (2) - um estudo mostra que, quando os dados são estratificados por macrorregiões e estados, existe tendência de declínio ou estabilização das taxas de mortalidade nas regiões com maior desenvolvimento socioeconômico e aumento dessas taxas nas regiões com menor desenvolvimento (3).

A mamografia é o principal exame de rastreamento do câncer de mama. A cobertura por mamografia acima de $70 \%$ pode reduzir em 20 a 30\% a mortalidade em mulheres com idade de 50 anos ou mais (4). Entretanto, estudos realizados no Brasil apontam coberturas insatisfatórias de mamografia na maioria dos estados das regiões Norte, Nordeste e Centro-Oeste (4-8). Entre as razões levantadas para essa situação, destacam-se o déficit de recursos humanos e a distribuição desigual de mamógrafos, com concentração nas capitais e centros urbanos (4). A distribuição inadequada desses equipamentos, sob viés da gestão pública, aguça as desigualdades, gerando sofrimento, mutilações e mortes (6-8). $\mathrm{O}$ não acesso à mamografia é destacado na literatura como um importante marcador de desigualdade em saúde (2), sendo relevante investigar o fenômeno à luz dos seus determinantes.

Um questionamento que pode ser levantado em vista desse cenário é se as políticas de incentivo para melhoria e expansão da atenção primária à saúde (APS), orientada pela Estratégia Saúde da Família (ESF), têm contribuído para o acesso das mulheres à realização da mamografia, frente, inclusive, ao acesso via saúde suplementar - setor que abriga os serviços de saúde prestados exclusivamente na esfera privada, detendo relação jurídica direta entre prestadores e usuários por meio de planos de saúde. Embora sem vinculação ao Sistema Único de Saúde (SUS) no Brasil, a saúde suplementar é regulada pelo Estado por meio da Agência Nacional de Saúde Suplementar (ANS) (9).

Diante do exposto, o presente estudo teve por objetivo avaliar a associação da ESF e da saúde suplementar na redução das desigualdades no acesso à mamografia no Brasil, evidenciando as áreas críticas dessa situação de saúde, a distribuição geográfica dos mamógrafos SUS e não SUS e as tendências de cobertura pelas duas modalidades de atenção. É importante destacar que, embora tenham sido conduzidas investigações que mensuram a cobertura de mamografia nos sistemas público e privado de atenção $(6-8,10,11)$, não foram encontrados estudos de amplitude nacional e que utilizam recursos cartográficos para avaliar a distribuição da cobertura por esse exame.

\section{MATERIAIS E MÉTODOS}

Foi realizado um estudo ecológico (12), considerando como unidades de análise as 27 unidades da federação. O Brasil possui 26 estados e um Distrito Federal agrupados em cinco macrorregiões: Norte (Rondônia, Acre, Amazonas, Roraima, Pará, Amapá, Tocantins), Nordeste (Maranhão, Piauí, Ceará, Rio Grande do Norte, Paraíba, Pernambuco, Alagoas, Sergipe, Bahia), Sudeste (Minas Gerais, Espírito Santo, Rio de Janeiro, São Paulo), Sul (Paraná, Santa Catarina, Rio Grande do Sul) e Centro-Oeste (Mato Grosso do Sul, Mato Grosso, Goiás e Distrito Federal) (13).

Segundo o Censo de 2010 do Instituto Brasileiro de Geografia e Estatística (IBGE), o Brasil possui população de 190755799 habitantes, Índice de Gini de 0,608 , produto interno bruto (PIB) de R\$ 3885847 002, renda média domiciliar per capita de $\mathrm{R} \$ 767,02$ e taxa de desemprego de 7,42 por habitante; $34,67 \%$ da população têm renda de menos de meio salário mínimo (13). Para o estudo, foram utilizadas variáveis referentes aos sistemas de saúde público e suplementar, indicadores de cobertura e desempenho do SUS e variáveis sociodemográficas.

A tabela 1 lista as variáveis obtidas do Departamento de Informática do SUS (DATASUS) que englobam informações do IBGE, do Cadastro Nacional de Estabelecimentos de Saúde (CNES), da ANS, do Departamento de Atenção Básica (DAB) e da Pesquisa Nacional de Saúde (PNS) referentes às 27 unidades federativas brasileiras. Avaliou-se a tendência temporal da proporção de cobertura populacional da ESF e a taxa de cobertura da saúde suplementar no período de 2005 a 2016. Sendo " $y$ " os valores da série temporal $e$ " $x$ " a escala de tempo, a reta de ajuste entre os pontos da série temporal cuja tendência se pretendeu estimar foi definida pela equação linear $\mathrm{y}=\mathrm{b}_{0}+\mathrm{b}_{1} \mathrm{x}$.

Para reduzir a heterogeneidade de variâncias dos resíduos da análise de regressão temporal, aplicou-se a transformação logarítmica dos valores de y (14). Assim, as taxas de cobertura (logarítmicas) foram calculadas pelo software Excel. A análise foi realizada com o programa estatístico Stata 13 usando o método de análise autorregrada conhecido como Prais-Winsten com vista à autocorreção temporal da primeira ordem dos resíduos.

O resultado dessa análise é a alteração percentual anual denominada taxa de incremento anual e seu respectivo intervalo de confiança de 95\% (IC95\%). A tendência é considerada decrescente se ambos os valores do intervalo de confiança são negativos, crescente se esses valores são positivos e estacionária quando o intervalo de confiança cruza o valor zero, ou seja, limite inferior e superior ficam com sinais opostos (14).

No presente estudo, a desigualdade em saúde é assumida como situações que implicam algum grau de injustiça, associadas a condições sociais que sistematicamentecolocam grupos em desvantagem quanto à possibilidade de usufruir de oportunidades e recursos em saúde (15). Para o estudo, foi selecionada como indicador de desigualdade a proporção de mulheres com idade de 50 a 69 anos que nunca realizaram mamografia. Esse indicador, extraído da PNS, foi adotado como variável dependente. A idade de 50 a 69 anos é a faixa etária prioritária para programas de rastreamento populacional do câncer de mama no Brasil (16). $\mathrm{O}$ indicador escolhido permite avaliar a adequação do acesso a mamografia, possibilitando a identificação de situações de desigualdade (2-5).

Com o propósito de verificar a relação da variável dependente com as variáveis independentes sociodemográficas e de serviços de saúde, foi utilizada a técnica de regressão linear múltipla para a seleção do melhor modelo explicativo (17). Para a seleção desse modelo, o algoritmo de stepwise e o critério de informação Akaike (AIC) foram usados para ajuste, sendo que para a escolha do modelo considerou-se o menor valor de AIC (17).

Em seguida foi realizado o teste de Shapiro-Wilk para testar a normalidade dos resíduos da regressão (17). A análise de regressão linear múltipla foi realizada no software Rstudio versão 3. Para incorporação das variáveis no modelo seguiuse a recomendação de Zuur et al. (18), com eliminação de outliers e certificação quanto a homogeneidade da variância, normalidade, colinearidade, relação entre $\mathrm{x}$ e $\mathrm{y}$, interação e independência do erro do modelo.

Como determina a legislação brasileira, por ter utilizado dados secundários 
TABELA 1. Variávieis (dependente e independentes) utilizadas no estudo sobre acesso à mamografia no Brasil, 2005 a 2016

\begin{tabular}{|c|c|c|}
\hline Fonte $^{\mathrm{a}}$ & Variável & Definição \\
\hline PNS & $\begin{array}{l}\text { Variável dependente: proporção de } \\
\text { mulheres de } 50 \text { a } 69 \text { anos de idade que } \\
\text { nunca realizaram exame de mamografia }\end{array}$ & $\begin{array}{l}\text { Número de mulheres de } 50 \text { a } 69 \text { anos de idade que nunca realizaram exame de mamografia dividido pelo total de } \\
\text { mulheres de } 50 \text { a } 69 \text { anos de idade, em determinado espaço geográfico no ano considerado. }\end{array}$ \\
\hline IBGE & Produto interno bruto (PIB) per capita & Valor do PIB municipal per capita, calculado como PIB municipal do ano dividido pela população do mesmo ano. \\
\hline IBGE & $\begin{array}{l}\text { Taxa de analfabetismo por ano segundo } \\
\text { unidade da federação }\end{array}$ & $\begin{array}{l}\text { Percentual de pessoas com } 15 \text { anos ou mais de idade que não sabem ler e escrever pelo menos um bilhete simples, } \\
\text { no idioma que conhecem, na população total residente da mesma faixa etária, em determinado espaço geográfico, } \\
\text { no ano considerado. }\end{array}$ \\
\hline IBGE & Renda média domiciliar per capita & Média das rendas domiciliares per capita das pessoas residentes em determinado espaço geográfico, no ano considerado. \\
\hline IBGE & População sem instalação sanitária & Proporção da população residente que não possui instalação sanitária. \\
\hline ANS & Taxa de cobertura por planos de saúde & $\begin{array}{l}\text { Razão entre o número de beneficiários e a população em uma área específica. Na ANS TABNETb , o cálculo é feito para } \\
\text { as grandes regiões, unidades da federação, capitais e regiões metropolitanas, por sexo e faixa etária. }\end{array}$ \\
\hline $\mathrm{DAB}$ & $\begin{array}{l}\text { Proporção de cobertura populacional } \\
\text { estimada por ESF }\end{array}$ & $\begin{array}{l}\text { Número de unidades de ESF multiplicado por } 3450 \text { (média de pessoas acompanhadas por unidade de ESF) divido pela } \\
\text { população em determinado espaço geográfico no ano considerado, com limitador de cobertura 100\%. }\end{array}$ \\
\hline CNES & $\begin{array}{l}\text { Cobertura de mamógrafos SUS e não } \\
\text { SUS por estabelecimento }\end{array}$ & $\begin{array}{l}\text { Número de mamógrafos SUS e não SUS pelo total de mamógrafos, segundo unidade da federação, no ano } \\
\text { considerado. }\end{array}$ \\
\hline
\end{tabular}

Fonte: DATASUS, 2017.

a ANS: Agência Nacional de Saúde; CNES: Cadastro Nacional de Estabelecimentos de Saúde; DAB: Departamento de Atenção Básica; IBGE: Instituto Brasileiro de Geografia e Estatística; PNS: Pesquisa Nacional de Saúde.

b TABNET: Informações em Saúde do DATASUS.

de acesso público, cujas informações de identificação individual não estão disponíveis, o estudo não precisou de aprovação por comitê de ética em pesquisa.

\section{RESULTADOS}

A figura 1 apresenta a renda domiciliar per capita em 2010, a porcentagem de mulheres de 50 a 69 anos que nunca haviam realizado mamografia e a comparação entre o crescimento da ESF e da saúde suplementar de 2005 a 2016 em cada uma das unidades da federação. As maiores rendas domiciliares per capita concentraram-se nas regiões CentroOeste e Sudeste, sendo os líderes Brasília, São Paulo e Rio de Janeiro, com rendas médias per capita de $\mathrm{R} \$ 1665,42$, $\mathrm{R} \$ 1$ 036,51 e R\$993,21, respectivamente. Quanto às mulheres de 50 a 69 anos que nunca realizaram mamografia, um alto percentual foi detectado em grande parte dos estados do Norte e Nordeste, especialmente Maranhão, Acre e Pará, com percentuais de $41,9 \%, 41,5 \%$ e $40,7 \%$, respectivamente.

A tabela 2 mostra a tendência temporal das taxas de cobertura da ESF e saúde suplementar para o período de 2005 a 2016. Todas as unidades da federação apresentaram tendência crescente da taxa de cobertura de saúde suplementar, exceto o Acre, que apresenta tendência estacionária. Em relação à cobertura pela ESF, Roraima, Tocantins, Maranhão, Piauí, Rio Grande do Norte e Paraíba apresentam tendência estacionária, enquanto os demais estados apresentaram cobertura crescente.

A figura 2A mostra proporção de mamógrafos do SUS em comparação aos mamógrafos não SUS por unidade da federação. O maior percentual de mamógrafos SUS foi observado em Roraima, Amazonas e Tocantins, com $87,5 \%, 84,7 \%$, 79,4\%, respectivamente; em contrapartida, Distrito Federal, Rio de Janeiro e Pará obtiveram 87,8\%, $66,8 \%$ e $60,3 \%$ de mamógrafos pelo sistema não SUS. A figura $2 \mathrm{~B}$ mostra a densidade de mamógrafos por 1000 mulheres de 50 a 69 anos. A maior densidade está em São Paulo, Minas Gerais, Rio de Janeiro e em toda costa litorânea brasileira.

Em relação ao modelo obtido pela regressão linear múltipla, observou-se que a variável dependente foi explicada por três das variáveis testadas, as quais alcançaram associação estatisticamente significativa. Segundo os resultados, as variáveis e seus parâmetros foram os seguintes: renda média domiciliar per capita $(\beta=-0,013$; IC95\% $=-0,027$ a $-0,001$; $P=0,041)$, cobertura pela ESF $(\beta=-0,242$; IC95\% $=-0,446$ a $-0,039 ; P=0,021)$ e cobertura pela saúde suplementar $(\beta=-0,804 ;$ IC95\% $=-1,157$ a $-0,452$; $P<0,001)$. Para os demais parâmetros, o AIC foi $=95,969, R^{2}=0,77$ e $P<0,001$. O teste de Shapiro-Wilk $(P=0,517)$ confirmou a normalidade dos resíduos do modelo de regressão linear proposto.

\section{DISCUSSÃO}

O presente estudo teve por objetivo avaliar a associação da ESF e da saúde suplementar com a redução das desigualdades de acesso à mamografia no Brasil, evidenciando as áreas críticas dessa situação de saúde, a distribuição geográfica dos mamógrafos SUS e não SUS e as tendências de cobertura pelas duas modalidades de atenção. Os achados revelaram uma tendência de crescimento tanto da ESF como da saúde suplementar, embora tenham sido observadas diferenças significativas entre as duas modalidades em todas as unidades federativas brasileiras. Nos últimos anos, houve grande expansão da ESF em todo o território nacional devido ao incentivo do Ministério da Saúde (19-21), todavia com ritmos diferentes entre os estados, como evidenciado no estudo.

Apesar dos estados de Roraima, Tocantins, Maranhão, Piauí, Rio Grande do Norte e Paraíba apresentarem tendência temporal estacionária quanto ao crescimento da cobertura da ESF, tais estados apresentaram altas coberturas dessa modalidade nos períodos de 2005 a 2016. Em regiões onde a cobertura já é elevada, torna-se desafiador sustentá-la (20). Outro fato que pode explicar a tendência estacionária é a dificuldade de fixação de profissionais de saúde, sobretudo médicos, em cidades do interior do Norte e Nordeste (22). Entretanto, o crescimento da ESF se reflete na diminuição 
FIGURA 1. A) Renda domiciliar per capita, B) porcentagem de mulheres de 50 a 69 anos que nunca haviam realizado mamografia e C) crescimento da ESF e da saúde suplementar, 2005 a 2016, Brasil

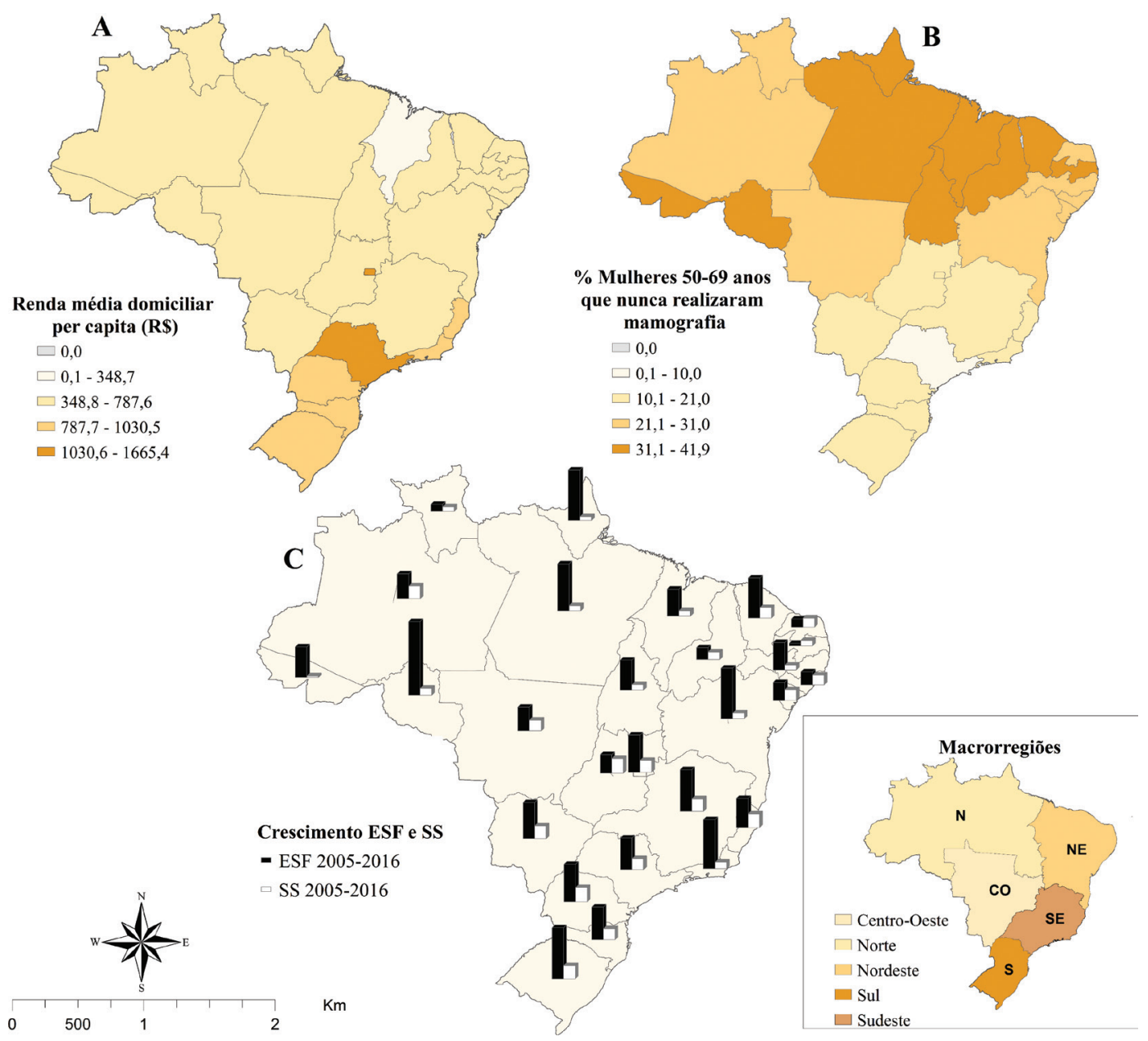

de taxas de internação hospitalar, melhoria de indicadores operacionais, diminuição de desigualdades em saúde e melhoria da qualidade de vida $(23,24)$. Outro resultado evidenciado no estudo é a expansão da saúde suplementar no Brasil. Conforme a literatura, observa-se que o crescimento da saúde suplementar se fortaleceu a partir da década de 1990, alcançando uma cobertura de $24,9 \%$ em 2016 (25).

Malta et al. (25), ao descreverem as coberturas de planos de saúde, relatam que as populações das regiões Sudeste e Norte têm a maior e a menor cobertura por planos de saúde, respectivamente, o que corrobora os achados deste estudo. Ain$\mathrm{da}$, segundo os resultados, o único estado que não apresentou tendência de crescimento da saúde suplementar foi o Acre, da região Norte. O aumento do número de planos de saúde na década de
1990 pode ser explicado pela conjuntura e estabilidade econômica naquele momento (26-28). Todavia, dado o cenário atual de desgaste financeiro e desemprego (na casa dos 12 milhões), essa situação pode ter se modificado.

Na comparação entre a porcentagem de mamógrafos SUS e não SUS por estabelecimento de saúde, tem-se que oito estados do Norte e Nordeste apresentam percentuais superiores a $60 \%$ de mamógrafos SUS em comparação com o Distrito Federal, Rio de Janeiro, São Paulo e Pará, que apresentaram percentuais superiores a $60 \%$ de mamógrafos não SUS. Segundo Oliveira et al. (5), houve maior financiamento do SUS para aquisição de mamógrafos, refletindo crescimento em escala nacional, bem como em aumento na região Norte. No Rio de Janeiro e no Distrito Federal, o alto percentual de mamógrafos não SUS provavelmente se relaciona à alta cobertura da saúde suplementar e à alta renda per capita nessas regiões.

O estudo mostrou que as regiões Norte e Nordeste, com menor densidade de mamógrafos, também apresentaram menor taxa de cobertura de mamografia, fenômeno que também foi observado por outros autores (2). No estudo, é nítida a má distribuição geográfica desses equipamentos, o que favorece a baixa cobertura da mamografia e compromete a acessibilidade geográfica das mulheres, especialmente as que residem em municípios pequenos e no interior, já que essas tecnologias estão concentradas nas regiões metropolitanas (6).

Há uma estimativa de que apenas $43,7 \%$ dos exames esperados segundo a necessidade foram realizados no SUS, o que deve colocar as autoridades sanitárias e gestores em alerta (6). Como a 
TABELA 2. Tendência temporal das taxas de cobertura pela Estratégia Saúde da Família e pela saúde suplementar, Brasil, 2005 a 2016 ${ }^{a}$

\begin{tabular}{|c|c|c|c|c|}
\hline \multirow{2}{*}{$\begin{array}{l}\text { Macrorregião/unidade } \\
\text { da federação }\end{array}$} & \multicolumn{2}{|c|}{ Estratégia Saúde da Família } & \multicolumn{2}{|c|}{ Saúde suplementar } \\
\hline & Coeficiente (IC95\%) & Tendência & Coeficiente (IC95\%) & Tendência \\
\hline \multicolumn{5}{|l|}{ Norte } \\
\hline Rondônia & $0,036(0,027$ a 0,045$)$ & Crescente & $0,022(0,004$ a 0,041$)$ & Crescente \\
\hline Acre & $0,011(0,003$ a 0,018$)$ & Crescente & $0,006(-0,001$ a 0,014$)$ & Estacionária \\
\hline Amazonas & $0,012(0,006$ a 0,018$)$ & Crescente & $0,032(0,011$ a 0,053$)$ & Crescente \\
\hline Roraima & $0,001(-0,008$ a 0,010$)$ & Estacionária & $0,023(0,009$ a 0,037$)$ & Crescente \\
\hline Pará & $0,034(0,019$ a 0,049$)$ & Crescente & $0,016(0,011$ a 0,020$)$ & Crescente \\
\hline Amapá & $0,018(0,007$ a 0,030$)$ & Crescente & $0,009(0,004$ a 0,015$)$ & Crescente \\
\hline Tocantins & $0,001(-0,027$ a 0,029$)$ & Estacionária & $0,026(0,023$ a 0,029$)$ & Crescente \\
\hline \multicolumn{5}{|l|}{ Nordeste } \\
\hline Maranhão & $0,005(-0,007$ a 0,017$)$ & Estacionária & $0,028(0,022$ a 0,034$)$ & Crescente \\
\hline Piauí & $-0,004(-0,026$ a 0,018$)$ & Estacionária & $0,029(0,023$ a 0,034$)$ & Crescente \\
\hline Ceará & $0,012(0,007$ a 0,019$)$ & Crescente & $0,024(0,019$ a 0,028$)$ & Crescente \\
\hline Rio Grande do Norte & $-0,004(0,027$ a 0,020$)$ & Estacionária & $0,018(0,009$ a 0,026$)$ & Crescente \\
\hline Paraíba & $0,000(-0,002$ a 0,002$)$ & Estacionária & $0,015(0,012$ a 0,018$)$ & Crescente \\
\hline Pernambuco & $0,009(0,008$ a 0,011$)$ & Crescente & $0,009(0,001$ a 0,018$)$ & Crescente \\
\hline Alagoas & $0,005(0,004$ a 0,006$)$ & Crescente & $0,026(0,019$ a 0,032$)$ & Crescente \\
\hline Sergipe & $0,004(0,002$ a 0,006$)$ & Crescente & $0,023(0,018$ a 0,029$)$ & Crescente \\
\hline Bahia & $0,019(0,016$ a 0,023$)$ & Crescente & $0,015(0,011$ a 0,019$)$ & Crescente \\
\hline \multicolumn{5}{|l|}{ Sudeste } \\
\hline Minas Gerais & $0,015(0,013$ a 0,017$)$ & Crescente & $0,015(0,005$ a 0,024$)$ & Crescente \\
\hline Espirito Santo & $0,015(0,013$ a 0,016$)$ & Crescente & $0,013(0,005$ a 0,021$)$ & Crescente \\
\hline Rio de Janeiro & $0,032(0,027$ a 0,038$)$ & Crescente & $0,006(0,002$ a 0,010$)$ & Crescente \\
\hline São Paulo & $0,026(0,022$ a 0,029$)$ & Crescente & $0,007(0,003$ a 0,011$)$ & Crescente \\
\hline \multicolumn{5}{|l|}{ Sul } \\
\hline Paraná & $0,016(0,014$ a 0,019$)$ & Crescente & $0,017(0,014$ a 0,021$)$ & Crescente \\
\hline Santa Catarina & $0,011(0,009$ a 0,013$)$ & Crescente & $0,011(0,003$ a 0,019$)$ & Crescente \\
\hline Rio Grande do Sul & $0,031(0,024$ a 0,038$)$ & Crescente & $0,016(0,007$ a 0,025$)$ & Crescente \\
\hline \multicolumn{5}{|l|}{ Centro-Oeste } \\
\hline Mato Grosso do Sul & $0,016(0,013$ a 0,019$)$ & Crescente & $0,018(0,013$ a 0,023$)$ & Crescente \\
\hline Mato Grosso & $0,009(0,006$ a 0,014$)$ & Crescente & $0,024(0,015$ a 0,033$)$ & Crescente \\
\hline Goiás & $0,008(0,007$ a 0,010$)$ & Crescente & $0,030(0,024$ a 0,035$)$ & Crescente \\
\hline Distrito Federal & $0,068(0,046$ a 0,090$)$ & Crescente & $0,010(0,001$ a 0,020$)$ & Crescente \\
\hline
\end{tabular}

distância implica uma importante barreira no acesso ao exame, uma alternativa pode ser a adoção de sistemas itinerantes. Entretanto, isso deve ser temporário, com data aprazada e estabelecida. Pelos resultados do estudo, entende-se que o acesso é resultado da oferta universal, da interiorização dos equipamentos e da distribuição da renda, tudo isso permeado por políticas de proteção sensíveis aos condicionantes observados no estudo (5). Na literatura, há evidências sobre determinantes do acesso à mamografia numa perspectiva geográfica (29), sobre conhecimento das mulheres em achar o exame necessário (29) e sobre a organização dos serviços de saúde para a oferta e demanda (30).
O presente estudo, a partir das estatísticas aplicadas, evidenciou uma associação significativa entre nunca ter realizado mamografia na idade de 50 a 69 anos, renda per capita e cobertura pela ESF e saúde suplementar. Tal resultado leva à constatação de que o acesso das mulheres à mamografia tem-se dado por meio das duas modalidades de atenção, achado relevante em termos de políticas públicas. Contudo, vale destacar a figura 2, que mostra algumas áreas críticas quanto ao acesso das mulheres nas regiões Norte e Nordeste, como Acre e Pará, onde, além de poucos, os mamógrafos estão em sua maioria vinculados ao sistema privado. Especificamente sobre o Acre, deve-se mencionar que é um dos estados com maior proporção de pessoas em condição de vulnerabilidade social extrema, sendo necessário avançar em termos de políticas públicas para a melhoria da justiça e da equidade.

Outro estado crítico em termos de acesso foi o Maranhão, onde, entretanto, percebe-se certa proporcionalidade na oferta de mamógrafos. Na maioria dos estados do Nordeste, o mamógrafo é oferecido pelo SUS, e nessas regiões observam-se coberturas elevadas de ESF. $\mathrm{O}$ acesso à mamografia foi prejudicado na Paraíba, talvez em decorrência de falhas na coordenação do cuidado da mulher na ESF ou na organização das redes de atenção à saúde.

De acordo com Oliveira et al. (5), houve um aumento importante da cobertura do exame de mamografia nas mulheres com baixa renda, por conta da expansão do número de mamógrafos financiados pelo SUS. Chor et al. (2) apontaram que entre as mulheres que tinham renda de até $\mathrm{R} \$ 100$ reais, $62 \%$ havia realizado mamografia. Em São Paulo, praticamente $76 \%$ das mulheres com essa renda teve a mamografia realizada; em Fortaleza, por sua vez, a proporção foi de $39 \%$, o que evidencia as desigualdades regionais. Adicionalmente, Chor et al. (2) concluem que usar o SUS, ter baixa escolaridade e baixa renda, ser solteira e idosa levam as mulheres a não buscarem a mamografia. Ao confrontar os resultados da presente investigação com os de Chor et al. (2), observa-se que a baixa renda também esteve associada ao não acesso. Todavia, os resultados não foram concordantes em termos do uso do SUS; a ESF aparece como um provável fator de proteção na presente investigação.

É importante destacar que no SUS há diferentes modalidades de APS, diferenças que devem ser consideradas, sob pena de viés ou erro de leitura. A modalidade tradicional (unidades básicas de saúde tracionais) está composta de médico generalista, ginecologista, pediatra, enfermeiros e auxiliares de enfermagem. Sua atenção organiza-se na lógica da demanda espontânea, mais inclinada ao atendimento das condições agudas ou agudizadas (31). A ESF, por sua vez, está fundamentada nos preceitos da Declaração de Alma-Ata (32), que propõem uma APS que responda de forma permanente, sistematizada e responsável à maior parte das necessidades de saúde de uma população, através do desenvolvimento de equipes multiprofissionais, 
FIGURA 2. A) Proporção de mamógrafos SUS e não SUS e B) densidade de mamógrafos por 1000 mulheres de 50 a 69 anos, Brasil, 2016

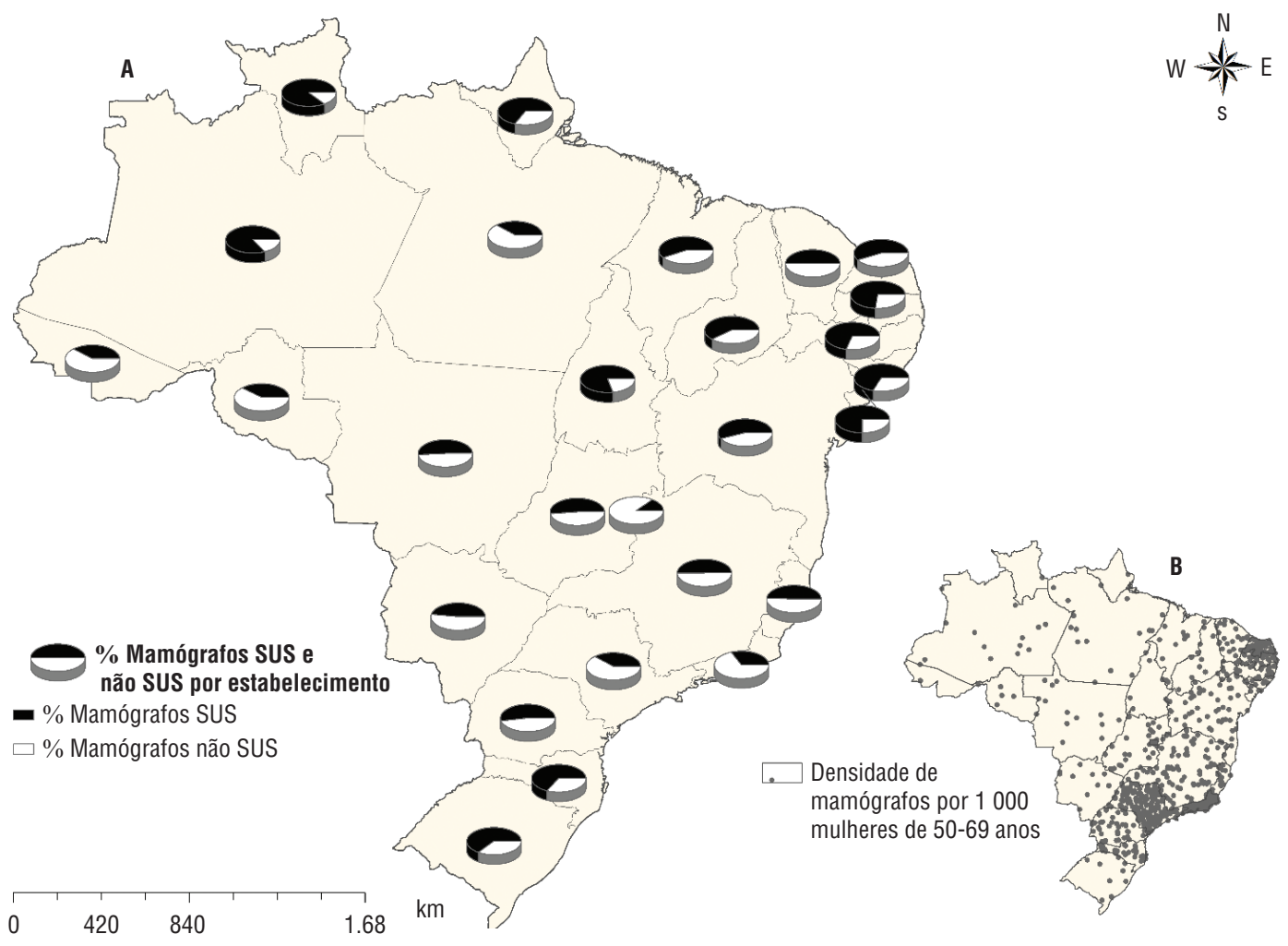

em território definido, que permite o levantamento das necessidades de saúde de forma organizada e resolutiva (33). Essa tem como diretrizes a integralidade e a equidade da atenção, a coordenação e a longitudinalidade do cuidado das famílias e pessoas (33); desse modo, talvez por não considerar essa distinção (entre unidades básicas de saúde tradicionais e ESF), Chor et al. (2) tenham encontrado que o uso do SUS estava associado ao não acesso à mamografia. Outro ponto sobre a saúde suplementar levantado por Azevedo e Silva et al. (11) é que ter plano privado de saúde é um dos fatores que mais se associaram positivamente com ter requisição para exame de mamografia. Contudo, morar nas regiões Norte e Nordeste reduz as chances de exame nessa modalidade.

Um importante achado deste estudo é que, embora o Norte e o Nordeste tenham maior cobertura de ESF, não é nessas regiões que se concentram os mamógrafos, talvez devido ao fato de que a disponibilidade e a oferta dos equipamentos siga a lógica do mercado, com concentração em áreas com maior poder de compra, como no estado de São Paulo. Os resultados indicam ainda que a maior parte dos mamógrafos nas regiões Sul e Sudeste está no setor privado, o que pode intensificar as desigualdades nessas regiões, especialmente naquelas populações que não têm planos privados e que residem em áreas com baixa cobertura de ESF. Fica claro que há uma fragilidade do Estado na regulação e distribuição desses insumos para a equidade em saúde. $\mathrm{O}$ teto dos gastos públicos (PEC 55), que congela o financiamento do SUS por 20 anos, compromete ainda mais a sustentabilidade de uma política de ESF universal (34).

Como limitações do presente estudo, a utilização de dados secundários pode ter gerado algum viés pela incompletude ou presença de informações ignoradas. Outra limitação é a heterogeneidade entre os estados, que não é captada quando se faz a opção por investigar as desigualdades por unidade federativa, havendo quase que uma homogeneização das informações no interior desse agregado. Para estudos futuros, seria oportuno o desenvolvimento de trabalhos qualitativos, visando a compreender as dificuldades das mulheres na busca por mamografia e suas experiências com aqueles serviços, assim como análises comparativas da ESF com outras modalidades de atenção, considerando as vivências e experiências sob o prisma da qualidade, segurança e satisfação.

\section{CONCLUSÃO}

O estudo possibilitou identificar as tendências de cobertura pela ESF e saúde suplementar no Brasil e como essas duas modalidades de atenção têm impactado o acesso à mamografia. Pelos achados, observou-se que a distribuição geográfica dos mamógrafos influencia diretamente a cobertura do exame de mamografia na faixa etária de 50 a 69 anos, principalmente nas regiões Norte e Nordeste, em que há menor densidade de mamógrafos.

A expansão da ESF e da saúde suplementar tem possibilitado o aumento do acesso às mamografias no Brasil, porém, em algumas regiões do país, o impacto da saúde suplementar é superior ao da 
ESF, principalmente porque os mamógrafos estão, em sua maioria, no sistema privado. Uma preocupação é que nessas regiões (Sul e Sudeste) também há uma baixa cobertura de ESF, o que faz com que as mulheres não tenham acesso a nenhuma das duas modalidades.

A desigualdade na distribuição de mamógrafos no Brasil perdura como entrave à equidade no acesso ao exame de mamografia. São urgentes medidas no sentido de reverter o quadro, sob Gomes da Silva. Mama. Rio de Janeiro: INCA; 2017. Disponível em: http:/ / www2. inca.gov.br/wps/wcm/connect/tiposdecancer/site/home/mama/cancer mama++. Acessado em 30 novembro 2017.

2. Chor D, Oliveira EXG, Melo ECP, Pinheiro RS, Carvalho MS. Desigualdade socioeconômica afeta a chance de realizar mamografia no Brasil. CMDSS 2011. Disponível em: http://cmdss2011.org/site/wp-content/uploads/2011/10/CMDSS-1110.1v1. pdf Acessado em 8 de agosto de 2018.

3. Guerra MR, Azevedo e Silva G, Nogueira MC, Leite ICG, Oliveira RVC, Cintra JRD, et al. Sobrevida por câncer de mama e iniquidade em saúde. Cad Saude Publica. 2015;31(8):1673-84.

4. Lages RB, Oliveira GP, Simeão Filho VM, Nogueira FM, Teles JBM, Vieira SC. Inequalities associated with lack of mammography in Teresina-Piauí-Brazil, 2010-2011. Rev Bras Epidemiol. 2012;15(4): 737-47.

5. Oliveira EXG de, Pinheiro RS, Melo ECP, Carvalho MS. Condicionantes socioeconômicos e geográficos do acesso à mamografia no Brasil, 2003-2008. Cienc Saude Colet. 2011;16(9):3649-64.

6. Xavier DR, Oliveira RAD de, Matos VP de, Viacava F, Carvalho C de C. Cobertura de mamografias, alocação e uso de equipamentos nas Regiões de Saúde. Saude Debate. 2016;40(110):20-35.

7. Villar VCFL, Souza CTV de, Delamarque EV, Seta MH De. Distribuição dos mamógrafos e dos exames mamográficos no estado do Rio de Janeiro, 2012 e 2013. Epidemiol Serv Saude. 2015;24(1):105-14.

8. Corrêa R da S, Freitas-Júnior R, Peixoto JE, Rodrigues DCN, Lemos ME da F, Marins LAP, et al. Estimativas da cobertura mamográfica no Estado de Goiás, Brasil. Cad Saude Publica. 2011;27(9): 1757-67.

9. Brasil. Lei $9961 / 2000$. Disponível em: http:/ / www.planalto.gov.br/ccivil_03/ LEIS/L9961.htm Acessado em setembro de 2018.

10. Souza CI de A, Araújo DS, Teles DA de F, Carvalho SGL de, Cavalcante KWM, Rabelo WL, et al. Factors related to nonadherence to mammography in a city of the pena de mais mulheres adoecerem e morrerem pelo câncer de mama no Brasil, o que é uma condição injusta e inaceitável.

Conflitos de interesse. Nada declarado pelos autores.

Agradecimentos. O presente trabalho foi realizado com apoio da Coordenação de Aperfeiçoamento de Pessoal de Nível Superior - Brasil (CAPES) - Código

\section{REFERÊNCIAS}

Brazilian Amazonian area: A populationbased study. Rev Assoc Med Bras. 2017; 63(1):35-42.

11. Azevedo e Silva G, Bustamante-Teixeira MT, Aquino EML, Tomazelli JG, dos Santos Silva I. Acesso à detecção precoce do câncer de mama no Sistema Unico de Saúde: uma análise a partir dos dados do Sistema de Informações em Saúde. Cad Saude Pública. 2014;30(7):1537-50.

12. Morgenstern H. Ecologic studies. Em: Rothman KJ, Lash TL, Greenland S. Modern epidemiology. Filadélfia: Lippincot Williams \& Wilkins; 2008. Pp. 512-531.

13. Instituto Brasileiro de Geografia e Estatística (IBGE). Censo Demográfico 2010. Rio de Janeiro: IBGE; 2013. Disponível em: http:/ / cidades.ibge.gov.br/xtras/home.php/ Acessado em 27 novembro 2017.

14. Antunes JLF, Cardoso MRA. Uso da análise de séries temporais em estudos epidemiológicos. Epidemiol Serv Saude. 2015;24(3): 565-76.

15. Barata RB. O que queremos dizer com desigualdades sociais em saúde? Em: Como e por que as desigualdades sociais fazem mal à saúde. Temas em Saúde. Rio de Janeiro: Editora Fiocruz; 2009. Pp. 11-21. Disponível em: http://books.scielo.org/ id/48z26/pdf/barata-9788575413913-02. pdf Acessado em 23 novembro 2017.

16. Migowski A, Stein AT, Ferreira CBT, Ferreira DMTP, Nadanovsky P. Guidelines for early detection of breast cancer in Brazil. I - Development methods. Cad Saude Publica. 2018;34(6):e00116317.

17. Hair Jr. JF, Anderson RE, Tatham RL, Black WC. Análise multivariada de dados. $6^{\mathrm{a}} \mathrm{ed}$. Porto Alegre: Bookman; 2009.

18. Zuur AF, Leno NE, Elphick CS. A protocol for data exploration to avoid common statistical problems. Methods Ecol Evol. 2010;1:3-14.

19. Fausto MCR, Giovanella L, Mendonça MHM de, Fonseca HMS, Lima JG. A posição da Estratégia Saúde da Família na rede de atenção à saúde na perspectiva das equipes e usuários participantes do PMAQ-AB 2012. Saude Debate. 2014; 38(spe):13-33.

20. Malta DC, Santos MAS, Stopa SR, Vieira JEB, Melo EA, Reis AAC dos. A cobertura da Estratégia de Saúde da Família (ESF) de Financiamento 001 - e do Conselho Nacional de Desenvolvimento Científico e Tecnológico (CNPq) pela concessão da bolsa produtividade em pesquisa (Processo 305236/2015-6).

Declaração. As opiniões expressas no manuscrito são de responsabilidade exclusiva dos autores e não refletem necessariamente a opinião ou política da RPSP/PAJPH ou da Organização Pan-Americana da Saúde (OPAS). no Brasil, segundo a Pesquisa Nacional de Saúde, 2013. Cienc Saude Colet. 2016;21(2): 327-38.

21. Brasil, Ministério da Saúde, Departamento de Atenção Básica do Ministério da Saúde. Histórico de cobertura. Disponível em: http:/ / dab.saude.gov.br/historico_cobertura_sf/historico_cobertura_sf_relatorio. php Acessado em 25 novembro 2017.

22. Linhares PHA, Lira GV, Albuquerque IMN. Avaliação do arograma de melhoria do acesso e qualidade no estado do Ceará. Saude Debate. 2014;38(spe):195-208.

23. Starfield B. Atenção primária: equilíbrio entre necessidades de saúde, serviços e tecnologia. Edição Brasileira. Brasília: UNESCO/Ministério da Saúde; 2002. Disponível em: https://www.nescon.medicina.ufmg.br/biblioteca/imagem/0253.pdf Acessado em setembro de 2018.

24. Kringos DS, Boerma WG, Bourgueil Y, Cartier T, Hasvold T, Hutchinson A, et al. The European primary care monitor: structure, process and outcome indicators. BMC Fam Pract. 2010;11(1):81.

25. Malta DC, Stopa SR, Pereira CA, Szwarcwald CL, Oliveira M, Reis AC dos. Cobertura de planos de saúde na população brasileira, segundo a Pesquisa Nacional de Saúde, 2013. Cienc Saude Colet. 2017;22(1):179-90.

26. Ziroldo RR, Gimenes RO, Júnior CC. A importância da saúde suplementar na demanda da prestação dos serviços assistenciais no Brasil. Mundo Saude. 2013;37(2): 216-21.

27. Santos FP dos, Malta DC, Merhy EE. A regulação na saúde suplementar: uma análise dos principais resultados alcançados. Cienc Saude Colet. 2008;13(5): 1463-75.

28. Santos IS, Ugá MAD, Porto SM. O mix público-privado no Sistema de Saúde Brasileiro: financiamento, oferta e utilização de serviços de saúde. Ciecn Saude Colet. 2008;13(5):1431-40.

29. Khan-Gates JA, Ersek JL, Eberth JM, Adams SA, Pruitt S. Geographic access to mammography and its relationship to breast cancer screening and stage at diagnosis: a systematic review. Womens Health Issues. 2015;25(5):482-93. 
30. Cruz-Jiménez L, Torres-Mejía G, MoharBetancourt A, Campero L, Ángeles-Llerenas A, Ortega-Olvera C, et al. Factors associated with ever use of mammography in a limited resource setting. A mixed methods study. Int J Qual Health Care. 2018;30(7):520-9.

31. Elias PE, Ferreira CW, Alves MCG, Cohn A, Kishima V, Escrivão Junior A et al. Atenção Básica em Saúde: comparação entre PSF e UBS por estrato de exclusão social no município de São Paulo. Cienc Saude Coletiva. 2006;11(3):633-41.

32. Pires-Alves FA, Cueto M. The Alma-Ata Decade: the crisis of development and international health. Cienc Saude Coletiva. 2017;22(7):2135-44.

33. Oliveira MAC, Pereira IC. atributos essenciais da atenção Primária e a estratégia Saúde da família. Rev Bras Enferm. 2013;66(esp):158-64.
34. Rossi P, Dweck E. Impactos do Novo Regime Fiscal na saúde e educação. Cad Saude Publica. 2016;32(12):e0019431.

Manuscrito recebido em 14 de dezembro de 2017 Aceito em versão revisada em 15 de agosto de 2018.
ABSTRACT

Family Health Strategy, private health care, and inequalities in access to mammography in Brazil
Objective. To evaluate the association between access to mammography and coverage by the Family Health Strategy (FHS) and supplementary (private) health insurance.

Method. An ecological study was performed with data obtained from the Unified Health System Data Processing Department (DATASUS). Time trends were analyzed using the Prais-Winsten method, using the Brazilian federal units (states) as units of analysis. Multiple linear regression was used to investigate the relationship between the dependent variable - women aged 50 to 69 years who had never had a mammogram - and the independent variables (coverage by the FHS or supplementary health insurance and socioeconomic aspects).

Results. Acre was the only Brazilian state for which a growth trend in supplementary health coverage was not observed. Roraima, Tocantins, Maranhão, Piauí, Rio Grande do Norte, and Paraíba showed a stable trend for FHS coverage; all other federal states showed an increase in coverage. A significant association was observed between never having had a mammogram at 50 to 69 years of age and two variables: mean per capita income and FHS and supplementary health coverage $\left(\mathrm{R}^{2}=0.77 ; \mathrm{P}<0.001\right)$.

Conclusion. Unequal access to mammography is a reality in Brazil. Both supplementary private health care and the FHS have helped to improve health care accessibility for Brazilian women.

Keywords Primary health care; Family Health Strategy; supplemental health; mammography; health status disparities; Brazil. 
RESUMEN

\section{Estrategia de Salud Familiar, salud suplementaria y desigualdad en el acceso a la mamografía en Brasil}

Objetivo. Evaluar la asociación entre el acceso a la mamografía en Brasil y la cobertura prestada por la Estrategia de Salud Familiar (ESF) y por la salud suplementaria.

Métodos. Se realizó un estudio ecológico con datos obtenidos del Departamento de Informática del Sistema Único de Salud (DATASUS). La tendencia de la serie temporal fue analizada mediante el método de Prais-Winsten utilizando como unidades de análisis las entidades federativas brasileñas. Para investigar la relación entre la variable dependiente - mujeres de 50 a 69 años que nunca se habían realizado una mamografía- y las independientes, de cobertura por la ESF o salud suplementaria y las variables socioeconómicas, se realizó un análisis de regresión lineal múltiple.

Resultados. Acre fue el único estado que no presentó una tendencia creciente para la cobertura por la salud suplementaria. Roraima, Tocantins, Maranhão, Piauí, Rio Grande do Norte y Paraíba presentaron una tendencia estacionaria para la cobertura por la ESF, mientras que las otras entidades federativas mostraron una cobertura en ascenso. Se observó una asociación significativa entre el hecho de nunca haberse realizado una mamografía entre los 50 y los 69 años y las variables renta media per cápita, cobertura por la ESF y la salud suplementaria $\left(R^{2}=0,77\right.$; $\mathrm{P}<0,001)$.

Conclusión. En Brasil, la desigualdad en el acceso a la mamografía es una realidad. Tanto la salud suplementaria como la Estrategia de Salud Familiar han contribuido a mejorar el acceso de estas mujeres a la mamografía.

Palabras clave Atención primaria de salud; Estrategia de Salud Familiar; salud complementaria; mamografía; disparidades en el estado de salud; Brasil. 